\title{
The Predictors of Moral Sensitivity Among Physicians
}

\author{
Nada Alyousefi (D) ${ }^{1,2}$ \\ Aljouhara Alibrahim' \\ Haifaa Taleb' \\ Lama Alotaibi ${ }^{1}$ \\ Leena Alrahmah' \\ Noorah Aldubaib (D) \\ Shahad Aljebreen' \\ Norah Alrowais ${ }^{1,2}$ \\ Jamal Aljarallah ${ }^{1,2}$ \\ 'Department of Family and Community \\ Medicine, College of Medicine, King Saud \\ University, Riyadh, Saudi Arabia; \\ ${ }^{2}$ Department of Family and Community \\ Medicine, King Saud University Medical \\ City, King Saud University, Riyadh, Saudi \\ Arabia
}

Purpose: The continually advancing nature of health care has improved the quality of care provided to patients. However, it has also resulted in complex ethical issues healthcare providers face in Saudi Arabia. Literature concerning healthcare workers' moral sensitivity in Saudi Arabia is limited. This study aims to estimate moral sensitivity among physicians and determine the factors that influence it.

Participants and Methods: A descriptive cross-sectional study was carried out among physicians working at a tertiary hospital. The Moral Sensitivity Questionnaire (MSQ) developed by Kim Lützén was used. The lowest score that can be obtained from the MSQ is 30 , and the highest score is 210 . Low scores demonstrate high ethical sensitivity, and high scores indicate low ethical sensitivity.

Results: A total of 253 physicians participated in the study. The mean score of moral sensitivity was $90.6 \pm 19.6$. There is a significant difference in the overall moral sensitivity in relation to age $(P=0.049)$. There are significant differences in the Moral conflict dimension according to age $(P=0.002)$, parental status $(P=0.011)$, being a member of an ethical committee $(P=0.025)$, years of experience $(P=0.002)$, clinical ranking $(P<0.001)$, and previous training in bioethics $(P=0.029)$. There were significant differences in the Relational orientation dimension with the clinical ranking $(P=0.038)$ and specialty $(P=$ 0.038). Membership of an ethical committee is a significant variable in the Benefit dimension $(P=0.028)$. Correlation coefficients between the overall moral sensitivity score and its dimensions were Autonomy $(\mathrm{r}=0.68)$, Practice $(\mathrm{r}=0.69)$, and Holistic approach $(\mathrm{r}=0.69)$. Physicians who previously had training in bioethics $(\beta 2.37, \mathrm{P}=0.022)$ and physicians who worked with clinical ethics committee $(\beta 2.66, \mathrm{P}=0.008)$ were more likely to score better in Moral conflict dimension.

Conclusion: Implementing ethical training for medical students and physicians will help raise their moral sensitivity levels, thereby enhancing how they deal with ethical dilemmas. Keywords: moral sensitivity, MSQ, ethics, physicians, bioethics

\section{Introduction}

The continually advancing nature of health care has improved the quality of care provided to patients. However, it has also resulted in the rise of complex ethical issues. ${ }^{1}$ Ethics and moral sensitivity are significant concepts in health care. Ethics could be defined as moral rules and values that offer professionals guidance in making decisions and delivering the best care possible. ${ }^{2}$

In daily practice, physicians have to decide on a course of action from multiple equally challenging alternatives; that is precisely why it is fundamental to analyze the ethical dimensions of a situation. ${ }^{3}$ Resolving and preventing ethical dilemmas requires moral sensitivity, ${ }^{4}$ which could be defined as recognizing an ethical issue
Correspondence: Nada Alyousefi Department of Family and Community Medicine, College of Medicine, King Saud University, Riyadh, II362, Saudi Arabia Tel +966II4670836

Email nalyousefi@ksu.edu.sa 
and understanding the ethical decision's impact on the patient's behalf. ${ }^{5}$ It is an important capacity to discern moral problems from other problems. ${ }^{6}$ Training, as shown in some studies, can help in establishing moral sensitivity. ${ }^{7-9}$ However, in a scoping review, Dalla Nora et al found that personal factors had the greatest impact on the development of moral sensitivity. ${ }^{10}$ It can be maintained by displaying the appropriate behaviors that follow the professional codes of ethics. ${ }^{8}$

Moral sensitivity evaluates the situation from multiple perspectives and considers each action's possible effectthis internal analysis aids in deciding the proper course of action. ${ }^{11}$ Professionals with poor moral sensitivity may fail to recognize existing moral situations, and therefore, fail to take appropriate action. ${ }^{11}$ The degree of morality is what guides physicians in making ethically sound judgments. ${ }^{8}$ There are, however, potential factors that may influence the decision-making process, such as socio-demographic characteristics, years of experience, prior ethical education, and specialty. ${ }^{8,12}$

Ethical issues in health care are discussed extensively in literature worldwide. ${ }^{13}$ One of the most common issues was that physicians felt pressured by patients and their families, leading to unwarranted investigations and treatments. On the other hand, the lack of adequate resources has led to the withdrawal of necessary treatments. ${ }^{14}$ This poses a significant challenge to the patient-physician relationship. ${ }^{15}$ Khosravani et al found that nurses who had high moral sensitivity had good communication skills. ${ }^{16}$ Moreover, increased ethical sensitivity is associated with diminished moral distress among healthcare professionals. ${ }^{17}$ This means that improving moral sensitivity scores among healthcare professionals might improve the quality of patient care.

Although bioethics teaching is implemented in medical schools worldwide and is supported by law workplace regulations, the reported moral sensitivity scores in literature were suboptimal. ${ }^{3,18,19}$ Furthermore, literature concerning healthcare workers' moral sensitivity in Saudi Arabia is limited. Only one publication was found of a study aimed to explore the psychometric properties of the Arabic-translated version of the moral sensitivity questionnaire among a sample of Saudi nursing students. ${ }^{20}$

Healthcare providers in Saudi Arabia currently face ethically challenging situations such as patient rights, equity of resources, and patient confidentiality. ${ }^{21}$ Estimating the level of moral sensitivity among physicians and its correlating factors may help implement strategies for physicians to improve their decision-making capacity, thereby improving the quality of care and providing ethically-based professional care. The current study aims at 1) Estimating the level of moral sensitivity among physicians and 2) Determining the factors that may influence their moral sensitivity.

\section{Materials and Methods}

This descriptive cross-sectional study was performed from September 2019 to April 2020 at a tertiary hospital in Riyadh, Saudi Arabia. The target population of the study consisted of 487 physicians.

The sample size was calculated by using the equation $\mathrm{n}=\mathrm{Z} 2 \alpha \mathrm{S} 2 / \mathrm{d} 2$. Twenty percent was added to the sample size to adjust for the non-responses that might happen. The final sample size was $\mathrm{N}=253$. A pilot study was done on twenty physicians before starting the study. It was not included in the sample. It aims to assess the survey's clarity. It appeared to be well understood by the participants.

The inclusion criteria entailed physicians working at King Saud University Medical City (KSUMC) for more than one year. Physicians were approached and asked to participate in the study at clinics, break rooms, ground meetings, rounds, and teaching sessions. A convenient sampling method was used.

The questionnaire had two parts. The first part consisted of eleven questions about the socio-demographic characteristics of the physicians. The second part was the tool to measure the moral sensitivity score, which is the Moral Sensitivity Questionnaire (MSQ) developed by Kim Lützén. The revised English version of the questionnaire is a valid instrument for assessing moral sensitivity; ${ }^{6}$ it was used in several studies involving nurses and physicians, mainly in psychiatric practice and nursing schools. ${ }^{8,20,22}$ A copy of MSQ was provided by Çetin and $\mathrm{Cimen}^{23}$ with their consent to use it in this study.

It is a seven-point Likert scale involving 30 items arranged under six subscales. ${ }^{1}$ Those were: (1) Autonomy indicates respecting the patient's choices. It consists of seven items (minimum score 7, maximum score 49 points). (2) Holistic approach refers to preventing harm to the patient and protecting their integrity. It consists of five items (minimum score 5, maximum score 35 points). (3) Moral conflict, meaning the encountered internal ethical conflicts. It consists of three items (minimum score 3, maximum score 21 points). (4) Relational orientation is the healthcare professional's concern for 
how actions affect the relationship with the patient. (5) Beneficence refers to acts that are motivated by doing what is believed to be best for the patient. (6) Practice refers to contemplating the ethical dimension while choosing an action or practice. Each of the last three dimensions consists of four items (minimum score 4, maximum score 28 points). Additional three statements of MSQ are not included in any subscale. ${ }^{1,6,23}$

The statements in the questionnaire are evaluated with a score between 1 and 7. Score 1 expresses high sensitivity with "complete agreement" and score 7 expresses low sensitivity with "complete disagreement." The lowest score that can be obtained from the MSQ is 30 , and the highest score is 210 . Low scores demonstrate high ethical sensitivity, and high scores demonstrate low ethical sensitivity.

The statistical package for social sciences (SPSS, version 24) was used for data analysis. Categorical variables were presented as frequencies and percentages. Numerical variables were presented as mean $\pm \mathrm{SD}$. $t$-test and ANOVA were used to compare the mean moral sensitivity score and its dimensions between the categorical variable level. Tukey pairwise comparison was used as a post hoc procedure to identify the groups' differences. A test with a p-value $<0.05$ was considered statistically significant.

Cronbach's alpha coefficient was used to analyze the reliability of the data collected. It was found to be (0.81). Results acquired from previous studies and this study signify that the scale is reliable. Significant variables $(p<0.05)$ were included in regression analyses to examine the predictors of moral sensitivity. The absolute value of Pearson correlationshowed no evidence of multicollinearity.

Ethical approval was obtained from the institutional review board of the college of medicine, King Saud University. A written consent indicating the purpose of the study and the participant's right to withdraw at any time without any obligation towards the study team was obtained from each participant.

\section{Results}

A total of 253 physicians participated in the study. About fifty-two percent (51.8\%) were females, the majority of the respondents were in the $25-30$ age group (45.8\%), and the least was in the $46-50$ age category (3.6\%). The mean age was $35.3( \pm \mathrm{SD}, 9.8)$.

The majority were Saudi (84.2\%), more than half of them were married (56.5\%), and (52.6\%) did not have children. Most physicians were trained locally after graduation $(79.1 \%)$, and most of them worked for five years or less $(59.3 \%)$. Only a few of them are current members of an ethical committee (3.2\%) and previous members (3.6\%). Regarding the clinical ranking, the majority of physicians were residents (45.5\%). Only $16.6 \%$ of participants attended multiple bioethics courses. Table 1 shows the characteristics of the participants.

The moral sensitivity overall score and its dimension were calculated to determine physicians' level of moral sensitivity. Table 2 shows the mean score of overall moral sensitivity $(90.6 \pm 19.6)$. Of all the dimensions of moral sensitivity, $25 \%$ of participants scored (78) or less, and $75 \%$ of participants scored (101.5) or less. The mean score of each dimension is shown in Table 2.

Table 3 shows the moral sensitivity level in relation to the demographic data of the study participants. Age was found to have an association with moral sensitivity level $(P=0.049)$. The lowest score was for the physicians' age category of fifty years or more, ie, higher moral sensitivity. It was also significant for the Moral conflict dimension $(P=0.002)$. Having children was not significantly associated with the moral sensitivity level except for the Moral conflict component. The specialty was associated significantly with the Relational orientation dimension $(P=0.038)$.

Physicians who have more than 15 years of experience had the highest moral sensitivity score $(93 \pm 19.3)$, ie, as the years of experience increase, the moral sensitivity decreases. However, the difference was not significant, except for the Moral conflict dimension $(P=0.002)$. Moral conflict and Relational orientation were all significantly affected by clinical ranking $(P<0.001)$ and $(P=0.043)$, respectively.

Current members of ethical committees had the highest moral sensitivity (10.4 \pm 2$)$. This association was significant for the Moral conflict dimension $(P=0.025)$ and Benefit dimension $(P=0.028)$. Although there was no significant association between the previous ethical training and the level of moral sensitivity, it was founded that those who received more training in bioethics significantly scored better in the Moral conflict dimension $(P=0.029)$ than those who did not attend courses.

Table 4 showed the correlation between the overall Moral sensitivity score and its dimensions, which showed moderate correlation with the following dimensions: (Autonomy; $\mathrm{r}=0.68$ ), (Practice; $\mathrm{r}=0.69$ ), and (Holistic approach; $\mathrm{r}=0.69$ ).

Table 5 shows the output of regression analysis. Two variables were found to explain $34.4 \%$ of the variance in Moral conflict dimension among participants. Physicians who previously had training in bioethics $(\beta 2.37, \mathrm{P}=0.022)$ 
Table I Characteristics of the Participants

\begin{tabular}{|c|c|c|c|c|c|c|c|}
\hline Variable & Category & $\mathbf{N}$ & $\%$ & Variable & Category & $\mathbf{N}$ & $\%$ \\
\hline \multirow[t]{2}{*}{ Gender } & Female & $|3|$ & 51.8 & \multirow{3}{*}{$\begin{array}{l}\text { Ethical Committee } \\
\text { Membership }\end{array}$} & Not a member of an ethical committee & 236 & 93.3 \\
\hline & Male & 122 & 48.2 & & A previous member of an ethical committee & 9 & 3.6 \\
\hline \multirow[t]{7}{*}{ Age } & $25-30$ & 116 & 45.8 & & A current member of an ethical committee & 8 & 3.2 \\
\hline & $31-35$ & 38 & 15 & \multirow[t]{8}{*}{ Clinical Rank } & Consultant & 80 & 31.6 \\
\hline & $36-40$ & 46 & 18.2 & & & & \\
\hline & $4 I-45$ & 15 & 5.9 & & Registrar and Fellow & 58 & 22.9 \\
\hline & $46-50$ & 9 & 3.6 & & & & \\
\hline & $51-55$ & 16 & 6.3 & & Resident & 115 & 45.5 \\
\hline & $>55$ & 13 & 5.1 & & & & \\
\hline \multirow[t]{2}{*}{ Nationality } & Saudi & 213 & 84.2 & & & & \\
\hline & Non-Saudi & 40 & 15.8 & & & & \\
\hline \multirow{2}{*}{$\begin{array}{l}\text { Marital } \\
\text { Status }\end{array}$} & Married & 143 & 56.5 & \multirow[t]{7}{*}{ Specialty } & Family Medicine, Internal Medicine, Pediatrics, & 174 & 68.8 \\
\hline & Not married & 110 & 43.5 & & Dermatology, Psychiatry & & \\
\hline \multirow{2}{*}{$\begin{array}{l}\text { Parental } \\
\text { Status }\end{array}$} & No children & 133 & 52.6 & & Surgery, Obstetrics, Gynecology & 46 & 18.2 \\
\hline & $\begin{array}{l}\text { Having } \\
\text { children }\end{array}$ & 120 & 47.4 & & $\begin{array}{l}\text { Emergency Medicine, Anesthesia, Intensive } \\
\text { Care Unit, Oncology }\end{array}$ & 25 & 9.9 \\
\hline \multirow[t]{3}{*}{$\begin{array}{l}\text { Place of } \\
\text { Training }\end{array}$} & $\begin{array}{l}\text { Local } \\
\text { program }\end{array}$ & 200 & 79.1 & & & & \\
\hline & Abroad & 50 & 19.8 & & Laboratory medicine, Radiology & 8 & 3.2 \\
\hline & Both & 3 & 1.2 & & & & \\
\hline \multirow{3}{*}{$\begin{array}{l}\text { Years of } \\
\text { Practice }\end{array}$} & $\leq 5$ years & 150 & 59.3 & \multirow{3}{*}{$\begin{array}{c}\text { Participants' Previous Training } \\
\text { in Bioethics }\end{array}$} & None & 6 & 2.4 \\
\hline & $6-15$ years & 75 & 29.6 & & $\mathrm{I}-2$ courses & 205 & 81 \\
\hline & $>15$ years & 28 & II.I & & $>2$ courses & 42 & 16.6 \\
\hline
\end{tabular}

Table 2 The Overall Score and Dimensions of Moral Sensitivity for the Study Participants

\begin{tabular}{|l|c|c|c|c|c|}
\hline Dimensions & Mean & SD & Median & QI & Q3 \\
\hline Moral sensitivity & 90.6 & 19.6 & 90 & $78^{*}$ & $101.5^{* *}$ \\
Autonomy & 19.6 & 5.1 & 19 & 16 & 22.5 \\
Holistic approach & 10.6 & 4.8 & 10 & 8 & 12 \\
Moral conflict & 12.8 & 4.4 & 12 & 10 & 16 \\
Relational orientation & 6.7 & 4.1 & 5 & 4 & 7.5 \\
Practice & 14.4 & 5.1 & 14 & 11 & 17 \\
Benefit & 13.0 & 4 & 13 & 10 & 16 \\
\hline
\end{tabular}

Notes: $* \mathrm{Q}$ I=the first quartile: $25 \%$ of participants scored 78 or less. ${ }^{*} \mathrm{Q} 3=$ the third quartile: $75 \%$ of participants scored 101.5 or less.

and physicians who worked with clinical ethics committees ( $\beta$ 2.66, $\mathrm{P}=0.008$ ), will be more likely to score better in Moral conflict dimension.

\section{Discussion}

Ethical decision-making plays a major role in clinical practice. Clinicians are faced with many moral challenges that need decisions, which might impose stress on them. The purpose of this study was to estimate the level of moral sensitivity among physicians at a tertiary hospital in Saudi Arabia and its determinants.

Moral sensitivity is an important attribute of healthcare workers and enables them to make appropriate ethical decisions. The overall level of moral sensitivity was moderate among most of the participants. This finding corresponds with what was found in previous studies. ${ }^{1,12,24}$ This could be explained by the lack of awareness of ethical issues and situations during clinical work. It may also be related to the nature of the clinical practice, where 
Table 3 The Overall Score and Dimensions of Moral Sensitivity in Correlation to the Demographic Data of the Study Participants

\begin{tabular}{|c|c|c|c|c|c|c|c|c|}
\hline \multicolumn{2}{|l|}{ Variable } & \multirow{2}{*}{\begin{tabular}{l}
\multicolumn{1}{c}{$\begin{array}{c}\text { Moral } \\
\text { Sensitivity }\end{array}$} \\
$91.9 \pm 18.4$ \\
$89.2 \pm 20.3$ \\
0.270
\end{tabular}} & \multirow{2}{*}{$\begin{array}{l}\text { Autonomy } \\
19.9 \pm 4.9 \\
19.3 \pm 5.3 \\
0.339\end{array}$} & \multirow{2}{*}{\begin{tabular}{l}
\multicolumn{1}{c}{$\begin{array}{c}\text { Holistic } \\
\text { Approach }\end{array}$} \\
$10.5 \pm 4.3$ \\
$10.8 \pm 5.1$ \\
0.694
\end{tabular}} & \multirow{2}{*}{$\begin{array}{l}\begin{array}{c}\text { Moral } \\
\text { Conflict }\end{array} \\
13.2 \pm 4.3 \\
12.5 \pm 4.6 \\
0.256\end{array}$} & \multirow{2}{*}{$\begin{array}{l}\begin{array}{c}\text { Relational } \\
\text { Orientation }\end{array} \\
6.6 \pm 3.9 \\
6.8 \pm 4.3 \\
0.755\end{array}$} & \multirow{2}{*}{$\begin{array}{l}\text { Practice } \\
14.9 \pm 5.1 \\
13.9 \pm 5.1 \\
0.114\end{array}$} & \multirow{2}{*}{$\begin{array}{l}\text { Benefit } \\
12.9 \pm 3.7 \\
13.1 \pm 4.3 \\
0.726\end{array}$} \\
\hline Gender & $\begin{array}{l}\text { Female } \\
\text { Male } \\
\text { P-value }\end{array}$ & & & & & & & \\
\hline Age & $\begin{array}{l}25-30 \\
31-35 \\
36-40 \\
41-45 \\
46-50 \\
51-55 \\
>55 \\
\text { P-value }\end{array}$ & $\begin{array}{l}88.2 \pm 19.5 \\
93.9 \pm 22.6 \\
88.8 \pm 13.9 \\
99.9 \pm 18.5 \\
81.2 \pm 26.7 \\
99.1 \pm 17.5 \\
81.2 \pm 26.7 \\
0.049 *\end{array}$ & $\begin{array}{l}19.7 \pm 4.9 \\
19.4 \pm 5.2 \\
18 \pm 4.3 \\
20.1 \pm 6.7 \\
18.1 \pm 6.8 \\
22.8 \pm 5.3 \\
18.1 \pm 6.8 \\
0.052\end{array}$ & $\begin{array}{l}10.5 \pm 4.1 \\
10.8 \pm 5.5 \\
9.9 \pm 3.8 \\
13.6 \pm 7.7 \\
8.8 \pm 2.8 \\
11 \pm 4.4 \\
8.8 \pm 2.8 \\
0.160\end{array}$ & $\begin{array}{l}11.9 \pm 4.2 \\
12.7 \pm 4.1 \\
13.2 \pm 4.5 \\
14 \pm 2.8 \\
12.1 \pm 6.5 \\
16.1 \pm 4.6 \\
12.1 \pm 6.5 \\
0.002\end{array}$ & $\begin{array}{l}6.9 \pm 3.9 \\
8.1 \pm 5.8 \\
5.7 \pm 2.5 \\
6.5 \pm 3.7 \\
4.9 \pm 1.6 \\
5.9 \pm 3.5 \\
4.9 \pm 1.6 \\
0.145\end{array}$ & $\begin{array}{l}13.9 \pm 4.7 \\
15.1 \pm 5.7 \\
14.5 \pm 5 \\
17.9 \pm 4.9 \\
13.2 \pm 7.6 \\
14.6 \pm 5.8 \\
13.2 \pm 7.6 \\
0.113\end{array}$ & $\begin{array}{l}12.5 \pm 3.9 \\
14.6 \pm 4.4 \\
13.5 \pm 4.3 \\
12.8 \pm 3.1 \\
11.1 \pm 4.1 \\
13.7 \pm 2.8 \\
12.6 \pm 4 \\
0.082\end{array}$ \\
\hline Nationality & $\begin{array}{l}\text { Saudi } \\
\text { Non-Saudi } \\
\text { P-value }\end{array}$ & $\begin{array}{l}90.3 \pm 19.3 \\
92 \pm 19.7 \\
0.626\end{array}$ & $\begin{array}{l}19.6 \pm 5.1 \\
19.3 \pm 5.4 \\
0.689\end{array}$ & $\begin{array}{l}10.5 \pm 4.3 \\
11.4 \pm 6.7 \\
0.304\end{array}$ & $\begin{array}{l}12.8 \pm 4.4 \\
13.1 \pm 4.7 \\
0.752\end{array}$ & $\begin{array}{l}6.7 \pm 4 \\
7 \pm 4.7 \\
0.669\end{array}$ & $\begin{array}{l}|4.3 \pm 5 .| \\
15.1 \pm 5.4 \\
0.336\end{array}$ & $\begin{array}{l}13 \pm 4 \\
13.2 \pm 4.1 \\
0.839\end{array}$ \\
\hline $\begin{array}{l}\text { Marital } \\
\text { status }\end{array}$ & $\begin{array}{l}\text { Married } \\
\text { Not married } \\
\text { P-value }\end{array}$ & $\begin{array}{l}91.2 \pm 20.1 \\
89.9 \pm 18.4 \\
0.589\end{array}$ & $\begin{array}{l}19.7 \pm 5.3 \\
19.4 \pm 4.9 \\
0.710\end{array}$ & $\begin{array}{l}10.6 \pm 5.3 \\
10.6 \pm 3.9 \\
0.991\end{array}$ & $\begin{array}{l}13.2 \pm 4.6 \\
12.3 \pm 4.2 \\
0.116\end{array}$ & $\begin{array}{l}6.6 \pm 4 \\
6.8 \pm 4.2 \\
0.715\end{array}$ & $\begin{array}{l}\mid 4.2 \pm 5.3 \\
\mid 4.6 \pm 4.9 \\
0.523\end{array}$ & $\begin{array}{l}13.2 \pm 3.9 \\
12.8 \pm 4.1 \\
0.402\end{array}$ \\
\hline $\begin{array}{l}\text { Parental } \\
\text { Status }\end{array}$ & $\begin{array}{l}\text { No children } \\
\text { Having Children } \\
\text { P-value }\end{array}$ & $\begin{array}{l}89.4 \pm 18.5 \\
91.9 \pm 20.3 \\
0.306\end{array}$ & $\begin{array}{l}19.4 \pm 4.9 \\
19.8 \pm 5.3 \\
0.536\end{array}$ & $\begin{array}{l}10.8 \pm 4.2 \\
10.5 \pm 5.3 \\
0.695\end{array}$ & $\begin{array}{l}12.2 \pm 4 \\
13.6 \pm 4.8 \\
0.011^{*}\end{array}$ & $\begin{array}{l}6.9 \pm 4 \\
6.5 \pm 4.2 \\
0.515\end{array}$ & $\begin{array}{l}\mid 4.5 \pm 4.9 \\
\mid 4.3 \pm 5.4 \\
0.743\end{array}$ & $\begin{array}{l}12.8 \pm 4 \\
13.3 \pm 4 \\
0.255\end{array}$ \\
\hline $\begin{array}{l}\text { Place of } \\
\text { Training }\end{array}$ & $\begin{array}{l}\text { Local program } \\
\text { Abroad } \\
\text { Both } \\
\text { P-value }\end{array}$ & $\begin{array}{l}90.4 \pm 19.7 \\
90.8 \pm \mid 8.2 \\
100.7 \pm \mid 3.4 \\
0.659\end{array}$ & $\begin{array}{l}19.7 \pm 5.1 \\
19 . \pm 5.1 \\
20.3 \pm 5 \\
0.698\end{array}$ & $\begin{array}{l}10.5 \pm 4.6 \\
11.2 \pm 5.3 \\
7.7 \pm 0.6 \\
0.357\end{array}$ & $\begin{array}{l}12.7 \pm 4.3 \\
13.3 \pm 5.1 \\
17 \pm 2 \\
0.183\end{array}$ & $\begin{array}{l}6.8 \pm 4.2 \\
6.3 \pm 4 \\
6.3 \pm 1.5 \\
0.687\end{array}$ & $\begin{array}{l}\mid 4.3 \pm 4.9 \\
\mid 4.5 \pm 5.8 \\
\mid 9 \pm 7.8 \\
0.29 \mid\end{array}$ & $\begin{array}{l}13.1 \pm 4.1 \\
12.7 \pm 3.6 \\
13.3 \pm 3.1 \\
0.844\end{array}$ \\
\hline $\begin{array}{l}\text { Years of } \\
\text { Practice }\end{array}$ & $\begin{array}{l}\leq 5 \text { years } \\
6-15 \text { years } \\
>15 \text { years } \\
\text { P-value }\end{array}$ & $\begin{array}{l}89.2 \pm 20.4 \\
92.5 \pm 16.9 \\
93 \pm 19.3 \\
0.378\end{array}$ & $\begin{array}{l}19.4 \pm 5 \\
19.3 \pm 5 \\
21.2 \pm 5.9 \\
0.207\end{array}$ & $\begin{array}{l}10.7 \pm 4.8 \\
10.5 \pm 5 \\
10.8 \pm 4.1 \\
0.964\end{array}$ & $\begin{array}{l}12.2 \pm 4.3 \\
13.4 \pm 4.6 \\
15.1 \pm 4.2 \\
0.002 *\end{array}$ & $\begin{array}{l}7.2 \pm 4.4 \\
6.3 \pm 3.7 \\
5.3 \pm 2.8 \\
0.043\end{array}$ & $\begin{array}{l}14.2 \pm 4.8 \\
15 \pm 5.7 \\
14.1 \pm 5 \\
0.447\end{array}$ & $\begin{array}{l}12.7 \pm 4.1 \\
13.9 \pm 3.9 \\
12.5 \pm 3.5 \\
0.069\end{array}$ \\
\hline $\begin{array}{l}\text { Clinical } \\
\text { Ranking }\end{array}$ & $\begin{array}{l}\text { Consultant } \\
\text { Registrar or Fellow } \\
\text { Resident } \\
\text { P-value }\end{array}$ & $\begin{array}{l}92.3 \pm 16.9 \\
93.7 \pm 22 \\
87.9 \pm 19.4 \\
0.118\end{array}$ & $\begin{array}{l}20 \pm 5.2 \\
19 \pm 5.4 \\
19.6 \pm 4.9 \\
0.534\end{array}$ & $\begin{array}{l}10.4 \pm 4.8 \\
11.2 \pm 5.7 \\
10.5 \pm 4.2 \\
0.599\end{array}$ & $\begin{array}{l}\mid 4.1 \pm 4.4 \\
\mid 3.7 \pm 4.9 \\
\mid 1.5 \pm 3.9 \\
<0.00 \mid *\end{array}$ & $\begin{array}{l}5.8 \pm 3.2 \\
7.4 \pm 5 \\
7 \pm 4.1 \\
0.038^{*}\end{array}$ & $\begin{array}{l}14.4 \pm 5.5 \\
15.4 \pm 5.4 \\
13.9 \pm 4.6 \\
0.182\end{array}$ & $\begin{array}{l}13.2 \pm 3.7 \\
13.6 \pm 4.1 \\
12.7 \pm 4.1 \\
0.335\end{array}$ \\
\hline Specialty & $\begin{array}{l}\text { Family Medicine, Internal Medicine, } \\
\text { Pediatrics, Dermatology, Psychiatry } \\
\text { Surgery, Obstetrics, Gynecology } \\
\text { Emergency Medicine, Anesthesia, ICU, } \\
\text { Oncology } \\
\text { Laboratory medicine, radiology } \\
\text { P-value }\end{array}$ & $\begin{array}{l}89.4 \pm 16.5 \\
92.2 \pm 23.9 \\
98.7 \pm 24.5 \\
81.6 \pm 24.8 \\
0.070\end{array}$ & $\begin{array}{l}19.6 \pm 4.7 \\
19.6 \pm 5 \\
20.3 \pm 7.3 \\
16.9 \pm 5.9 \\
0.441\end{array}$ & $\begin{array}{l}10.3 \pm 3.8 \\
10.5 \pm 4.9 \\
13.1 \pm 7.7 \\
11.1 \pm 8.1 \\
0.055\end{array}$ & $\begin{array}{l}\mid 2.9 \pm 4.3 \\
|3 \pm 5 .| \\
\mid 3.5 \pm 4.2 \\
9.9 \pm 4.3 \\
0.243\end{array}$ & $\begin{array}{l}6.3 \pm 3.3 \\
7.5 \pm 5.2 \\
8.4 \pm 6.4 \\
\\
5.5 \pm 2.6 \\
0.038^{*}\end{array}$ & 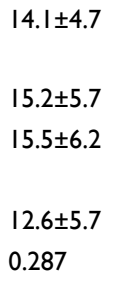 & $\begin{array}{l}12.9 \pm 3.9 \\
13.2 \pm 4.5 \\
13.9 \pm 4.5 \\
13.1 \pm 2.5 \\
0.697\end{array}$ \\
\hline $\begin{array}{l}\text { Ethical } \\
\text { Committee } \\
\text { Membership }\end{array}$ & $\begin{array}{l}\text { Not a member of an ethical committee } \\
\text { A previous member of an ethical } \\
\text { committee } \\
\text { A current member of an ethical } \\
\text { committee } \\
\text { P-value }\end{array}$ & $\begin{array}{l}90.4 \pm 19.6 \\
102.8 \pm 11.7 \\
82.6 \pm 11.1 \\
0.084\end{array}$ & $\begin{array}{l}19.5 \pm 5.2 \\
20.8 \pm 4.2 \\
20.4 \pm 3.5 \\
0.691\end{array}$ & $\begin{array}{l}10.6 \pm 4.8 \\
11.6 \pm 5.4 \\
10.3 \pm 1.8 \\
0.823\end{array}$ & $\begin{array}{l}12.8 \pm 4.5 \\
16.1 \pm 3.4 \\
10.4 \pm 2 \\
0.025\end{array}$ & $\begin{array}{l}6.7 \pm 4.1 \\
7.3 \pm 5.1 \\
5.8 \pm 2.4 \\
0.725\end{array}$ & $\begin{array}{l}14.4 \pm 5.1 \\
16.4 \pm 5.4 \\
11.8 \pm 5 \\
0.166\end{array}$ & $\begin{array}{l}13.0 \pm 4.1 \\
13.0 \pm 4.1 \\
11 \pm 2.4 \\
0.028^{*}\end{array}$ \\
\hline
\end{tabular}


Table 3 (Continued).

\begin{tabular}{|l|l|l|l|l|l|l|l|l|}
\hline \multicolumn{2}{|l|}{ Variable } & $\begin{array}{c}\text { Moral } \\
\text { Sensitivity }\end{array}$ & Autonomy & $\begin{array}{c}\text { Holistic } \\
\text { Approach }\end{array}$ & $\begin{array}{c}\text { Moral } \\
\text { Conflict }\end{array}$ & $\begin{array}{c}\text { Relational } \\
\text { Orientation }\end{array}$ & Practice & Benefit \\
\hline Previous & None & $94.3 \pm 14.4$ & $21.5 \pm 2.3$ & $11.2 \pm 3.7$ & $14.7 \pm 3.7$ & $6.9 \pm 4.4$ & $15.3 \pm 4.1$ & $13 \pm 4.1$ \\
Bioethics & $1-2$ courses & $93 \pm 15.6$ & $19.9 \pm 5.1$ & $10.6 \pm 5$ & $14.3 \pm 4.1$ & $5.9 \pm 2.6$ & $15 \pm 4.5$ & $13.2 \pm 3.6$ \\
Training & $>2$ courses & $90 \pm 20.2$ & $19.5 \pm 5.2$ & $10.6 \pm 4.8$ & $12.5 \pm 4.5$ & $5.5 \pm 1.4$ & $14.3 \pm 5.3$ & $11.7 \pm 2.9$ \\
& P-value & 0.599 & 0.583 & 0.955 & $0.029 *$ & 0.263 & 0.649 & 0.676 \\
\hline
\end{tabular}

Notes: $* \mathrm{P}<0.05$ was statistically significant.

Table 4 Correlation Coefficients Between Overall Moral Sensitivity Score and Its Dimensions

\begin{tabular}{|c|c|c|c|c|c|c|c|}
\hline $\begin{array}{l}\text { Pearson } \\
\text { Correlation }\end{array}$ & $\begin{array}{c}\text { Moral } \\
\text { Sensitivity }\end{array}$ & Autonomy & $\begin{array}{l}\text { Holistic } \\
\text { Approach }\end{array}$ & $\begin{array}{c}\text { Moral } \\
\text { Conflict }\end{array}$ & $\begin{array}{l}\text { Relational } \\
\text { Orientation }\end{array}$ & Practice & Benefit \\
\hline Moral Sensitivity & 1 & 0.68 & 0.69 & 0.56 & 0.55 & 0.69 & 0.54 \\
\hline Autonomy & & I & 0.53 & 0.18 & 0.44 & 0.30 & 0.22 \\
\hline Holistic Approach & & & 1 & 0.12 & 0.63 & 0.35 & 0.18 \\
\hline Moral Conflict & & & & 1 & -0.04 & 0.42 & 0.22 \\
\hline Relational Orientation & & & & & 1 & 0.21 & 0.20 \\
\hline Practice & & & & & & 1 & 0.27 \\
\hline Benefit & & & & & & & 1 \\
\hline
\end{tabular}

Table 5 Predictors of the Score of Moral Conflict Dimension Among Participants $(\mathrm{n}=253)$

\begin{tabular}{|l|c|c|c|}
\hline Predictor & $\begin{array}{c}\text { Regression } \\
\text { Coefficients }\end{array}$ & $\mathbf{t}$ & P-value \\
\hline Ethics Committee Membership & 0.161 & 2.655 & $0.008^{*}$ \\
Parental Status & 0.053 & 0.675 & 0.500 \\
Age & 0.155 & 1.398 & 0.163 \\
Clinical Rank & 0.172 & 1.863 & 0.064 \\
Years of Practice & 0.001 & 0.013 & 0.989 \\
Ethics Education and Courses & 0.161 & 2.370 & $0.022^{*}$ \\
\hline
\end{tabular}

Notes: Summary of model: $R=0.344 ; R^{2}=0.122$; model fit: $F=5.518 p<0.001$; statistically significant (Enter method). *Significant predictor.

physicians are busy and have to make several decisions simultaneously.

Moreover, physicians who practice in Arab and Muslim countries understand its culture. The family plays a major role in medical decisions and can interfere with patient autonomy. ${ }^{25}$ Interestingly, most ethical standards have roots in Islamic jurisprudence. For example, the patient's consent is essential in Islam, even if his condition is serious, as long as he or she is considered a competent adult. Although many physicians try to implement what they learned in medical ethics, they face many difficulties with some patients and families. Hence, they adopt different approaches that could contradict ethical standards. ${ }^{25}$ Fortunately, the situation is improved in
Saudi Arabia with governmental regulations in the healthcare system and having trained young generations trying to empower patients and implement ethical standards.

One of the main Moral Sensitivity Questionnaire (MSQ) dimensions is the interpersonal relationship, which focuses on a trusting relationship. ${ }^{5}$ It received the highest sensitivity in our study. This was concordant with the findings of Kırılmaz et al. ${ }^{12}$ This may be because the interpersonal relationship is highly valued in clinical practice and forms an important determinant of clinicians' success, and reflects professionalism.

There was no correlation between gender and the level of moral sensitivity. In contrast, a study determined that female nurses' moral sensitivities were higher than male nurses, with no significant difference between the groups. ${ }^{26}$ Lutzen et al showed significant differences in responses between males and females, ie, females had higher levels of moral sensitivity, especially in the "structuring moral meaning" dimension. ${ }^{19}$

The current study found an association between age and having a higher moral sensitivity level. This specific factor appears to vary tremendously between published studies. Different studies found that as age increases, morals sensitivity increases as well. ${ }^{26-28}$ A statistically significant difference was found between the age and the total mean score of the scale and autonomy subscale in the study of Tazegun and Celebioglu. ${ }^{26}$ Another study reported that when physicians get older, they tend to 
express more benevolence. ${ }^{27}$ The mean scores of the healthcare personnel aged 40 years or older were determined to be higher. ${ }^{28}$ A study on nurses found that as they progress in age, their moral sensitivity becomes higher. ${ }^{4}$

The parental status was significantly associated with higher moral sensitivity, which was similar to the finding of a study of nursing students. ${ }^{29}$ Another study found that ethical sensitivity levels were higher in participants who have children in only a holistic approach dimension. ${ }^{12}$ Another study reported a higher moral sensitivity score in the orientation subscale among nurses with children, ${ }^{1}$ similar to Tosun et al's study findings. ${ }^{28}$ The parental status was significant for the Moral conflict dimension.

There was no correlation between ethical education and the level of moral sensitivity. A similar finding was reported by Kurlmaz et al and Basak et al ${ }^{12,24}$ Basak et al ${ }^{24}$ found no relationship between receiving ethics education and the intensive care nurses' ethical sensitivity level. In contrast, some studies reported a higher score with more ethical education and training. ${ }^{1}$ A possible explanation is that most of those published studies in this regard were concerning students at nursing schools. ${ }^{1}$ It is important to know that studies reported significant differences between physicians and nurses in moral sensitivity, ${ }^{19}$ which makes it harder to compare. The current study found that the training in bioethics is a predictor only for moral conflict dimension score.

Physicians who have more than 15 years of experience were found to have the lowest moral sensitivity score. In comparison, physicians who have five years or less of experience have the highest moral sensitivity, more evident in the moral conflict dimension. A similar finding was reported by Kırılmaz et al $^{12}$ ie, ethical sensitivity was higher in those less than five years than 10-14 years and $\geq 15$ years $(P<0.05)$ in the conflict dimension. On the contrary, in a study conducted on nurses, a higher moral sensitivity was reported among those who had long years of work experience. ${ }^{1}$ An interesting finding by Nejadsarvari et $\mathrm{al}^{27}$ is that there was no significant correlation between moral sensitivity and years of work experience. One would expect that moral sensitivity will improve with experience as the workers' capability to deal with ethical issues will be better. A possible explanation for this contradiction could be that this is a form of ethical decay.

As for clinical positions, residents had the highest moral sensitivity, whereas consultants had the lowest. Moral conflict and Relational orientation were all significantly affected by clinical ranking. Experiencing ethically challenging decisions might affect the physicians' well-being, leading to burnout which is common among senior-level employees due to their role in the clinical setting. ${ }^{30}$

Relational orientation dimension score was significantly associated with the specialty. The highest moral sensitivity was in the laboratory medicine and radiology group, followed by internal medicine. The lowest Moral Sensitivity was in Emergency Medicine, Anesthesia, oncology, and intensive care unit (ICU) physicians. Similarly, in Nejadsarvari et $\mathrm{al}^{27}$ study, moral sensitivity among emergency medicine specialists was the lowest. In contrast to our study, Kirilmaz et $\mathrm{al}^{12}$ found that the moral sensitivity level of those working in ICU and operating rooms was higher than those working in polyclinics. Another study found that nurses' ethical sensitivity in primary health care was higher than nurses working in hospitals. $^{31}$

Furthermore, Çetin \& Cimen reported that dimension scores of autonomy and following the rules and total score were better in practitioners, while dimension scores of conflict were better in specialists. ${ }^{23}$ A possible explanation for our finding in relation to specialties could be that laboratory and radiology physicians are not exposed to ethical challenges than other specialties. This could be supported by Saarni et al's study where psychiatrists experienced the most ethically challenging decisions, followed by pulmonologists, internists, and neurologists. In contrast, it was least often by pathologists and laboratory physicians. ${ }^{13}$

Physicians who are members of the ethical committee had higher moral sensitivity levels in all dimensions except autonomy. The hospital ethics committees help and assist physicians who are dealing with moral difficulties in clinical practice. ${ }^{32}$ Hence, the members' educational level aid in improving the overall management of bioethical issues. In another study, Lasker et $\mathrm{al}^{33}$ stated that most ethical committee members had adequate knowledge and comprehension of bioethics. However, some members are still not completely aware of bioethical issues. ${ }^{33}$ Furthermore, a qualitative assessment can be conducted to explore this area.

One of the limitations of this study is that the Moral Sensitivity Questionnaire (MSQ) developed by Lützén ${ }^{6}$ does not have cut-off points. A lower score reflects higher ethical sensitivity. Additionally, no directly comparable study was published, which makes the interpretation difficult. The 
sample was well represented to our population of interest and relatively large, hence high in statistical power. As a result, generalizability is possible but is limited due to the cross-sectional study design. A multicenter study can add more information. A focus group, qualitative assessment for the senior physician, can add important details.

\section{Practical Implication}

Improving the moral sensitivity among physicians can help implement ethically-based professional care, hence improving the quality of patient care. Practical exercises regarding ethical decision-making and case discussion can increase moral sensitivity and, necessarily, physicians' well-being.

\section{Conclusion}

This study showed a moderate overall level of moral sensitivity. Significant differences were found in the dimensions of the MSQ scale with age, parental status, membership status of ethical committees, years of experience, clinical ranking, bioethics training, and specialty. It was determined that junior physicians had a higher level of moral sensitivity than senior physicians. Also, physicians who are members of the ethical committee had a higher moral sensitivity. Implementing ethical training for medical students, physicians in training, with particular attention to train the senior working physicians in different specialties with continuous professional developmental activities, will help raise their moral sensitivity levels, thereby enhancing how they deal with ethical dilemmas.

\section{Abbreviation}

MSQ, Moral Sensitivity Questionnaire.

\section{Data Sharing Statement}

The datasets generated and analyzed during the current study are available from the corresponding author on reasonable request.

\section{Ethics Approval and Consent to Participate}

Ethical approval was obtained from the institutional review board, College of Medicine, King Saud University (No. CMED305-F8, dated 6/11/2019). A written consent indicating the purpose of the study and the participant's right to withdraw at any time without any obligation towards the study team was obtained from each participant.

\section{Author Contributions}

All authors made a significant contribution to the work reported, whether that is in the conception, study design, execution, acquisition of data, analysis, and interpretation, or in all these areas; took part in drafting, revising, or critically reviewing the article; gave final approval of the version to be published; have agreed on the journal to which the article has been submitted; and agree to be accountable for all aspects of the work.

\section{Funding}

There is no funding to report.

\section{Disclosure}

The authors report no conflicts of interest in this work.

\section{References}

1. Tas Arslan F, Calpbinici P, Tas Arslan F, Calpbinici P. Moral sensitivity, ethical experiences and related factors of pediatric nurses: a cross- sectional, correlational study. Acta Bioeth. 2018;24(1):9-18. doi:10.4067/S1726-569X2018000100009

2. Yilmaz Sahin S, Iyigun E, Acikel C. Validity and reliability of a Turkish version of the modified moral sensitivity questionnaire for student nurses. Ethics Behav. 2015;25(4):351-359. doi:10.1080/ 10508422.2014.948955

3. Ersoy N, Gundogmus UN. A study of the ethical sensitivity of physicians in Turkey. Nurs Ethics. 2003;10(5):472-484. doi:10. 1191/0969733003ne6290a

4. Ertug N, Aktas D, Faydali A, Yalçin O. Ethical sensitivity and related factors of nurses working in the hospital settings/Sensibilidad ética y factores relacionados con el trabajo de enfermera/o en hospitales/ Sensibilidade ética e fatores relacionados com enfermeiros que trabalham em hospi. Acta Bioeth. 2014;20(2):265-270. doi:10.4067/ S1726-569X2014000200014

5. Lützén K, Evertzon M, Nordin C. Moral sensitivity in psychiatric practice. Nurs Ethics. 1997;4(6):472-482. doi:10.1177/09697330970 0400604

6. Lützén K, Dahlqvist V, Eriksson S, Norberg A. Developing the concept of moral sensitivity in health care practice. Nurs Ethics. 2006;13(2):187-196. doi:10.1191/0969733006ne837oa

7. Baykara ZG, Demir SG, Yaman S. The effect of ethics training on students recognizing ethical violations and developing moral sensitivity. Nurs Ethics. 2015;22(6):661-675. doi:10.1177/09697330 14542673

8. Akca NK, Simsek N, Arslan DE, Senturk S, Akca D. Moral sensitivity among senior nursing students in Turkey. Int J Caring Sci. 2017;10(2):1031-1039.

9. Bahrieni F, Azodi P, Hajivandi A, Jahanpour F. The effect of education in nurse's moral sensitivity. J Pharm Sci Res. 2017;9(10): 1817-1821.

10. Dalla Nora CR, Zoboli E, Vieira MM. Moral sensitivity of nurses assessed through scoping review. Cogitare Enferm. 2017;22(2): e47162. 
11. Rushton CH, Kaszniak AW, Halifax JS. A framework for understanding moral distress among palliative care clinicians. J Palliat Med. 2013;16(9):1074-1079. doi:10.1089/jpm.2012.0490

12. Kirilmaz H, Harun MA, Kahraman G. A research about the ethical sensitivity of healthcare professionals. Int J Health Sci. 2015;3(3):73-82.

13. Saarni SI, Halila R, Palmu P, Vanska J. Ethically problematic treatment decisions in different medical specialties. J Med Ethics. 2008;34 (4):262-267. doi:10.1136/jme.2007.020529

14. Saarni SI, Parmanne P, Halila R. Ethically problematic treatment decisions: a physician survey. Bioethics. 2008;22(2):121-129. doi:10.1111/j.1467-8519.2007.00608.x

15. Povar GJ, Blumen H, Daniel J, et al. Ethics in practice: managed care and the changing health care environment: medicine as a profession managed care ethics working group statement. Ann Intern Med. 2004;141 (2):131-136. doi:10.7326/0003-4819-141-2-200407200-00012

16. Khosravani M, Borhani F, Loghmani L, Mohsenpour M. Ethical sensitivity relationship with communication skills in Iranian nursing managers. Int J Pharm Res. 2018;10(3):143-147.

17. Sedghi Goyaghaj N, Zoka A, Mohsenpour M. Moral sensitivity and moral distress correlation in nurses caring of patients with spinal cord injury. Clin Ethics. 2021;1477750921994279. doi:10.1177/1477750 921994279

18. Borhani F, Abbaszadeh A, Hoseinabadi-Farahani MJ. Moral sensitivity and its dimensions in Iranian nursing students. J Med Ethics Hist Med. 2016;9:19.

19. Lutzen K, Johansson A, Nordstrom G. Moral sensitivity: some differences between nurses and physicians. Nurs Ethics. 2000;7 (6):520-530. doi:10.1177/096973300000700607

20. Bayoumy HMM, Halabi JO, Esheaba OM. Translation, cultural adaptation, validity and reliability of the moral sensitivity questionnaire for use in Arab countries. Saudi J Health Sci. 2017;6(3):151. doi:10.4103/sjhs.sjhs_97_17

21. Alkabba AF, Hussein GMA, Albar AA, Bahnassy AA, Qadi M. The major medical ethical challenges facing the public and healthcare providers in Saudi Arabia. J Family Community Med. 2012;19 (1):1-6. doi:10.4103/2230-8229.94003
22. Lützén K, Nordin C. The influence of gender, education and experience on moral sensitivity in psychiatric nursing: a pilot study. Nurs Ethics. 1995;2(1):41-50. doi:10.1177/096973309500200106

23. Cetin M, Cimen M. Assessing a group of physicians' ethical sensitivity in Turkey. Iran J Public Health. 2011;40(3):89-97.

24. Basak T, Uzun Ş, Arslan F. Investigation of the moral sensibility of intensive care nurses. Gulhane Med J. 2010;52:76-81.

25. Al-Bar MA, Chamsi-Pasha H. Contemporary Bioethics: Islamic Perspective. Springer Nature. 2015.

26. Tazegün A, Çelebioğlu A. Ethical sensitivity levels of pediatric nurses and the factors affecting this sensitivity. $J \mathrm{Dr}$ Behcet $U z$ Child Hosp. 2016;6(2):97-102. doi:10.5222/buchd.2016.097

27. Nejadsarvari N, Abbasi M, Borhani F, et al. Relationship of moral sensitivity and distress among physicians. Trauma Mon. 2015;20(2): e26075. doi:10.5812/traumamon.26075

28. Tosun H. Determination of sensitivities of physicians and nurses against ethical dilemmas experienced in health care practices; 2005.

29. Tuvesson H, Lützén K. Demographic factors associated with moral sensitivity among nursing students. Nurs Ethics. 2017;24(7): 847-855. doi:10.1177/0969733015626602

30. Shanafelt TD, Boone S, Tan L, et al. Burnout and satisfaction with work-life balance among US physicians relative to the general US population. Arch Intern Med. 2012;172(18):1377-1385. doi:10.1001/ archinternmed.2012.3199

31. González-de Paz L, Kostov B, Sisó-Almirall A, Zabalegui-Yárnoz A. A Rasch analysis of nurses' ethical sensitivity to the norms of the code of conduct. J Clin Nurs. 2012;21(19-20):2747-2760. doi:10.11 11/j.1365-2702.2012.04137.x

32. Hajibabaee F, Joolaee S, Cheraghi MA, Salari P, Rodney P. Hospital/ clinical ethics committees' notion: an overview. J Med Ethics Hist Med. 2016;9:17.

33. Lasker S, Hossain A, Shakoor M. Knowledge and attitude of Ethics Committee (EC) members regarding bioethics and structure \& function of EC in Bangladesh: a pilot study; 2019.
International Journal of General Medicine

\section{Publish your work in this journal}

The International Journal of General Medicine is an international, peer-reviewed open-access journal that focuses on general and internal medicine, pathogenesis, epidemiology, diagnosis, monitoring and treatment protocols. The journal is characterized by the rapid reporting of reviews, original research and clinical studies across all disease areas. The manuscript management system is completely online and includes a very quick and fair peer-review system, which is all easy to use. Visit http://www.dovepress.com/ testimonials.php to read real quotes from published authors. 